\title{
Developmental epileptic encephalopathy with hypomyelination and brain atrophy associated with PTPN23 variants affecting the assembly of UsnRNPs
}

\author{
Robert Smigiel ${ }^{1} \cdot$ Gerd Landsberg $^{2} \cdot$ Maximilian Schilling $^{2} \cdot$ Małgorzata Rydzanicz $^{3} \cdot$ Agnieszka Pollak $^{4}$. \\ Anna Walczak ${ }^{3} \cdot$ Anna Stodolak $^{1} \cdot$ Piotr Stawinski $^{3,4} \cdot$ Hanna Mierzewska $^{5} \cdot$ Maria M. Sasiadek $^{6}$ \\ Oliver J. Gruss ${ }^{2} \cdot$ Rafal Ploski $^{3}$
}

Received: 17 November 2017 / Revised: 20 April 2018 / Accepted: 26 April 2018 / Published online: 13 June 2018

(c) European Society of Human Genetics 2018

\begin{abstract}
PTPN23 encodes a ubiquitously expressed non-receptor type, catalytically inactive protein-tyrosine phosphatase found in all cells including neurons. Recently, we have identified PTPN23 in a cellular screen for the systematic identification of novel regulators of survival motor neuron (SMN) function in the assembly of splicing factors (Uridine-rich small nuclear ribonucleoproteins, UsnRNPs). Based on three families, recessive PTPN23 variants have been associated with human disease tentatively, without functional studies. Here, we describe a pediatric proband with severe developmental delay, epilepsy, cortical blindness, hypomyelination and brain atrophy on MRI. Whole exome sequencing and family study showed two novel PTPN23 variants, c.1902C >G (p.(Asn634Lys)) and c.2974delC (p.(Leu992Tyrfs*168)), in compound heterozygous state, which are predicted in silico to be damaging. When studying patient's fibroblasts we found similar expression of SMN but a dramatic reduction of cells displaying SMN accumulation in Cajal bodies (CB). SMN strongly accumulated in CB in more than 50\% of unrelated control cell fibroblasts as well as in fibroblasts from the parent carrying only the c.2974delC (p.(Leu992Tyrfs*168)) variant (predicted to cause loss-of-function). In contrast, only $22 \%$ of cells showed respective SMN accumulations in patient fibroblasts $\left(p=1.9-2.5 \times 10^{-7}\right)$ while showing a higher level of nucleoplasmic SMN. Furthermore, the remaining accumulations in patient cells displayed weaker SMN signals than control or heterozygous wt/c.2974delC (p.(Leu992Tyrfs*168)) fibroblasts. Our report provides the first description of the clinical phenotype of recessive PTPN23 variants with pathogenicity substantiated by a functional study.
\end{abstract} Ploski.

Electronic supplementary material The online version of this article (https://doi.org/10.1038/s41431-018-0179-2) contains supplementary material, which is available to authorized users.

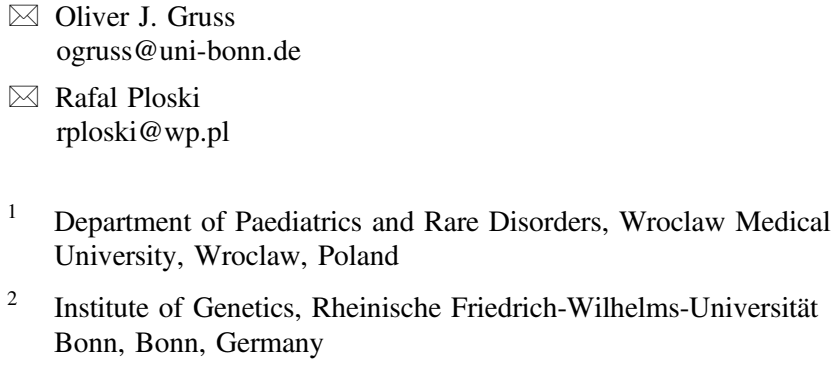

1 Department of Paediatrics and Rare Disorders, Wroclaw Medical University, Wroclaw, Poland

2 Institute of Genetics, Rheinische Friedrich-Wilhelms-Universität Bonn, Bonn, Germany

These authors are contributed equally: Oliver J. Gruss and Rafal

\section{Introduction}

PTPN23 encodes a ubiquitously expressed non-receptor type protein-tyrosine phosphatase found in all cells including neurons (http://www.proteinatlas.org). As predicted by its primary structure, it was demonstrated that PTPN23 does not display dephosphorylation activity on phospho-tyrosine residues and may, therefore, serve a

3 Department of Medical Genetics, Warsaw Medical University, Warsaw, Poland

4 Department of Genetics, Institute of Physiology and Pathology of Hearing, Warsaw, Poland

5 Department of Child and Adolescent Neurology, Institute of Mother and Child, Warsaw, Poland

6 Department of Genetics, Wroclaw Medical University, Wroclaw, Poland 
function of maintaining tyrosine phosphorylation patterns of substrate proteins [1]. Consistently, PTPN23 has been implicated in diverse cellular functions. As part of the ESCRT-I complex (endosomal sorting complex required for transport I (ref. [2])), it plays a role in sorting of endocytic, ubiquitinated cargos into multivesicular bodies (MVBs). Moreover, it was assigned a role in ciliogenesis [3] and as a negative regulator of Ras-mediated mitogenic activity [4]. In mice, homozygous deletion of PTPN23 is lethal around embryonic day 9.5 (ref. [5]), while heterozygous mice display higher tumor susceptibility for lung adenoma and B cell lymphoma indicating a role for PTPN23 as a tumor suppressor [6].

Recently, in an unbiased screen analyzing all human phosphatase gene products after RNAi-knockdown in human cells, PTPN23 was identified as a regulator of survival motor neuron complex (SMN) function in the assembly of UsnRNPs (U-rich small nuclear ribonucleoproteins) [7]. UsnRNPs are ubiquitous complexes consisting of RNA and protein moieties. Together with other protein complexes they form the macromolecular machineries (spliceosomes) that mediate pre-mRNA splicing [8]. Mutations in genes encoding UsnRNPs are associated with retinal dystrophies, hereditary acrofacial syndromes, and hematological malignancies [8]. Besides that, genetic defects in other components regulating splicing, such as HNRNPU [9] or HNRNPH2 (ref. [10]), were recently implicated in neurodevelopmental disorders/epileptogenesis.

The highly conserved SMN protein forms a complex (SMN complex) that, in all eukaryotes, assists the correct assembly of novel UsnRNPs in the cytoplasm, accompanies newly formed UsnRNPs into the nucleus and accumulates in nuclear substructures termed Cajal bodies (CB). Our screen for the identification of SMN regulators exploited the accumulation of SMN in nuclear $\mathrm{CB}$, which is generally correlated with the activity of human cells to assemble new UsnRNPs [11]. It revealed that knockdown of PTPN23 results in significantly reduced accumulation of SMN in CB indicating a role for PTPN23 in regulating the activity of SMN in UsnRNP biogenesis [7].

Recessive PTPN23 variants have been associated with human disease only tentatively, based on single families coming from large cohorts of clinically diverse patients with little details as to specific manifestations. Alazami et al. [12] described PTPN23 p.(Arg1332Leu) homozygosity in a child with global developmental delay, epilepsy and brain atrophy. Recently, Trujillano et al. [13] found a homozygous variant, p.(Met302 Val), in a proband with global developmental delay with regression and spasticity, microcephaly, seizures as well as brain atrophy. In both cases the patients were from consanguineous families and no functional studies were performed to validate the pathogenicity of the reported PTPN23 missense variants.
In the present study we provide a detailed description of a proband with novel compound heterozygous PTPN23 variants and a clinical phenotype consistent with previous reports. We substantiate the PTPN23 dysfunction by showing that patient's fibroblasts have altered SMN activity.

\section{Results}

\section{Clinical report}

The proband was the first child of a healthy nonconsanguineous Polish-Belarussian couple. The family history was non-contributory. The girl was born at 40 weeks of gestation by natural delivery after an uneventful pregnancy with the following birth parameters: weight $2320 \mathrm{~g}$ ( $300 \mathrm{~g}$ below 3 percentile), length $52 \mathrm{~cm}$ (50-90 percentile), OFC $30 \mathrm{~cm}$ ( $2 \mathrm{~cm}$ below 3 percentile), 9 points of Apgar scale at $1 \mathrm{~min}$. The newborn was treated because of intrauterine growth retardation, hypotonia, poor sucking, and feeding problems. TORCH infection was excluded. No congenital defects were noted after birth. Echocardiography examination showed foramen ovale.Developmental milestones were severely delayed from the newborn period. During the first three months of life hypotonia was observed later followed by severe spasticity. Epileptic seizures have been observed from 2 years of age. Electroencephalography (EEG) performed several times showed multifocal discharges. Treatment with vigabatrin led to no clinical improvement but its replacement with depakine and levetiracetam decreased the number of seizures and improved the EEG result. The child was deaf and blind with ophthalmological examination showing atrophy of optic nerves and abnormal visual evoked potentials (VEP). Central thyroid insufficiency was observed as well. Tandem mass spectrometry (MS/MS), gas chromatography-mass spectrometry (GC/MS) assays as well as amino acids profile revealed no abnormalities excluding main inborn errors of amino acid, organic acid, and mitochondrial fatty acid metabolism. Lactic acid, CK, and NH3 levels were in the normal range.

Before whole exome sequencing (WES) the following genetic tests were performed yielding normal results: karyotype (normal female), a methylation test in direction of Angelman syndrome and MLPA assay with telomeric probes, SETBP1 gene sequencing.

Array CGH (SurePrint G3 CGH v2, $8 \times 60 \mathrm{~K}$, analyzed on G2600D SureScan Microarray Scanner System, Agilent) revealed microduplication of $756 \mathrm{~kb}$ at $\mathrm{Xp} 22.33$; the same microduplication was found in the healthy mother. The duplicated region observed in aCGH has not been reported as pathogenic in the literature. Agilent SurePrint G3 CGH 
Fig. 1 External appearance of the patient, note microcephaly and protruding tongue
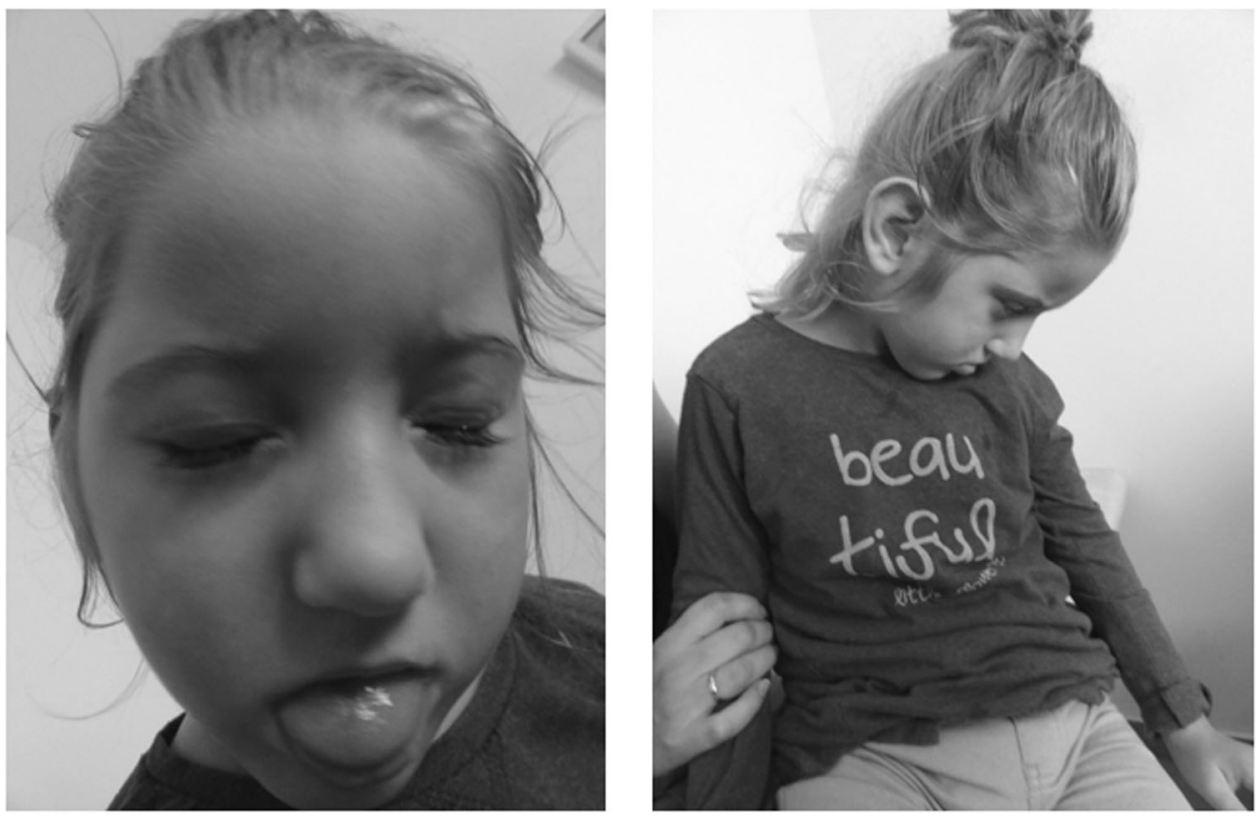
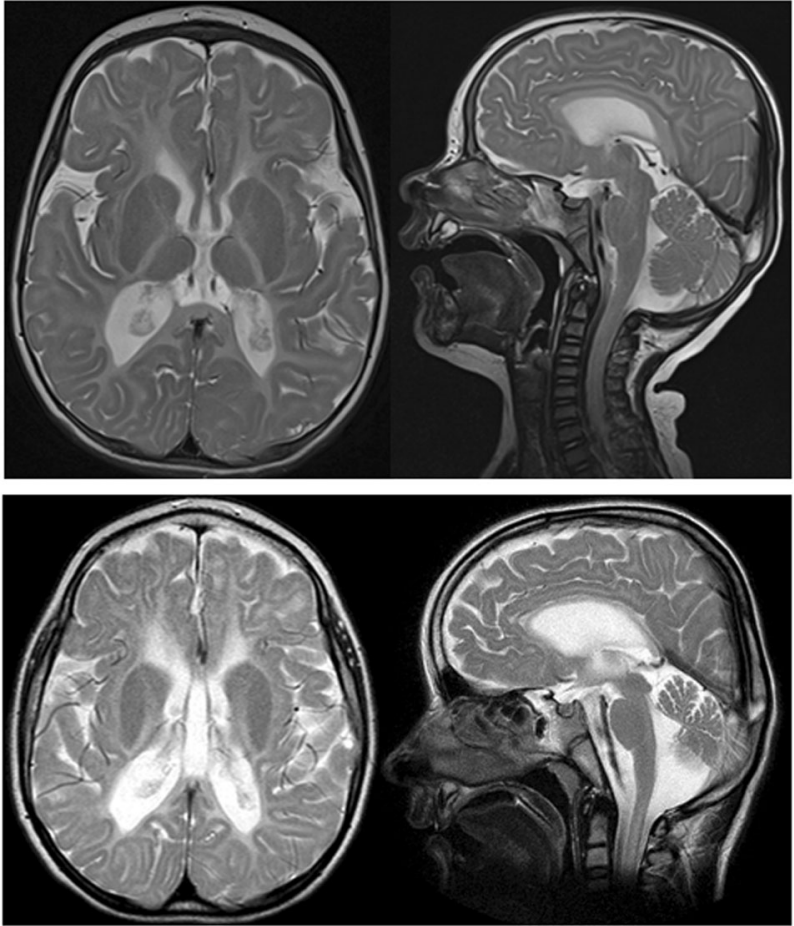

Fig. 2 Brain MRI (T2) at 4 months (top) and 4 years (bottom) of age. Top left: axial section — global lack of myelin, also in posterior limb of internal capsule. Top right: sagital section-lack of myelination, normal size of unmyelinated corpus callosum. Bottom left: corticosubcortical atrophy and global lack of myelin, decreased volume and rarefaction of white matter; secondary widening of lateral ventricles and preserved cavum septi pellucidi; normal signal and volume of basal ganglia. Bottom right: sagittal section-lack of myelination and thinning of corpus callosum, atrophy of cerebellum array is dedicated for analysis of 498 genomic regions with highest clinical importance according to International Standards for Cytogenomic Arrays (ISCA). The ISCA regions are analyzed with $\sim 20 \mathrm{~kb}$ resolution while remaining regions are covered with 1 probe per $60 \mathrm{~kb}$ which allows to detect aberrations $\geq 300 \mathrm{~kb}$.

At the last follow-up, at the age of 6 years, the proband's weight was $13 \mathrm{~kg}$ ( $3 \mathrm{~kg}$ below $3 \mathrm{rd}$ percentile), length 115 $\mathrm{cm}(25-50$ percentile) and head circumference $46 \mathrm{~cm}(3 \mathrm{~cm}$ below 3rd percentile). Apart from microcephaly and protruding tongue no dysmorphic features were observed (Fig. 1). There was severe spasticity of all four limbs, no eye contact, and no verbal communication. The proband could not walk, sit or speak.

Brain MRIs were repeated several times from infancy to 4 years of life and showed a lack of supratentorial and subtentorial myelination (Fig. 2). With time, a progressive cortico-subcortical atrophy of the brain with markedly decreasing volume and rarefaction of white matter were visible, as well as secondary to atrophy a widening of lateral ventricles and cavum septum pellucidum. Basal ganglia had normal signal and volume. Atrophy of the cerebellum was also visible. The overall picture suggested progressive encephalopathy with hypomyelination.

\section{Genetic study}

Bioinformatics analysis of whole exome sequencing (WES) data, revealed two variants potentially affecting function, both in PTPN23 NG_051056.1(NM_015466.3):c. [1902C>G];[2974del] (Table 1). 
Table 1 PTPN23 variants in proband

Fig. 3 PTPN23 sequencing results in proband and her parents. Whole exome sequencing (WES) results in the proband were visualized using Integrative Genomics Viewer (IGV)

\begin{tabular}{|c|c|c|c|c|c|}
\hline cDNA level & Protein & Gene & $\begin{array}{l}\text { Reference } \\
\text { sequence }\end{array}$ & $\begin{array}{l}\text { Chromosomal } \\
\text { position }\end{array}$ & Zygosity \\
\hline c. $1902 C>G$ & p.(Asn634Lys) & PTPN23 & \multirow{2}{*}{$\begin{array}{l}\text { NG_051056.1; } \\
\text { NM_015466.3 }\end{array}$} & $\operatorname{chr} 3: 047451011-\mathrm{C}>\mathrm{G}$ & heterozygous \\
\hline c.2974delC & p.(Leu992Tyrfs*168) & PTPN23 & & $\begin{array}{l}\text { chr3:047452260- } \\
\text { TC }>\text { T }\end{array}$ & heterozygous \\
\hline
\end{tabular}

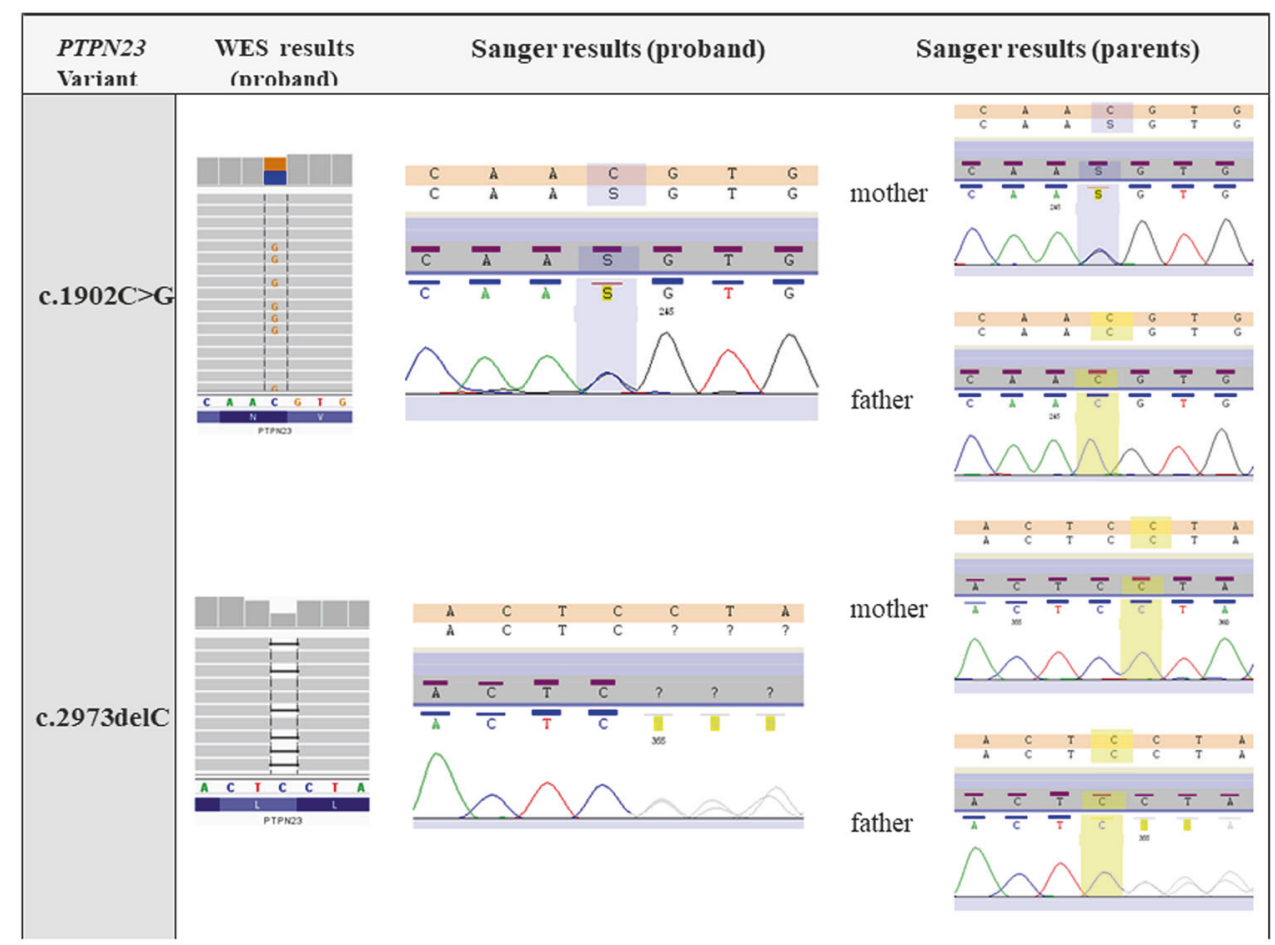

Family study using Sanger sequencing confirmed the presence of both heterozygous variants in the PTPN23 in the proband and demonstrated that they were located on separate chromosomes (compound heterozygous state, Fig. 3). The c.1902C $>\mathrm{G}$ (p.(Asn634Lys)) variant was inherited from the mother, whereas the c.2974delC (p. (Leu992Tyrfs*168)) variant was inherited from the father.

The PTPN23 c.1902C >G (p.(Asn634Lys)) missense variant is likely to be damaging to protein function as assessed by CADD (score of 11.5) as well as Polyphen2 and Mutation Taster (predicted as damaging by both programs). The c.2974delC (p.(Leu992Tyrfs*168)) 1 bp deletion variant represents a frameshift variant, therefore it is very likely to be damaging. Neither of these PTPN23 variants have been reported in gnomAD database (http://gnomad.broa dinstitute.org), nor our own database including over 1,000 WES samples from the Polish population.

All variants considered as potentially disease-causing are listed in Supplemental Data, Table 1. FASTQ and VCF files from WES are available on request to qualified researchers.
Notably, no plausible rare variants in other genes than $P T P N 23$, including $S M N 1 / 2$, were found in WES.

\section{Functional studies}

Given the role of PTPN23 in regulation of SMN function we decided to evaluate SMN localization and function in patient's cells. We isolated and cultivated patient fibroblasts and compared them with fibroblasts from a healthy nonrelated control person and the patient's father carrying the c.2974delC (p.(Leu992Tyrfs*168)) variant. Of note, the control cell types expressed PTPN23 to a similar degree while the expression of full length PTPN23 was consistently even higher in patient cells (Fig. 4). All cells displayed comparable SMN levels, however, a slower migrating, possibly phosphorylated form of SMN was absent in patient's cells (Fig. 4, see *). To estimate the cellular activity of SMN, we tested the accumulation of SMN in CB by indirect immunofluorescence [7]. In control fibroblasts as well as in fibroblasts isolated from the father, 


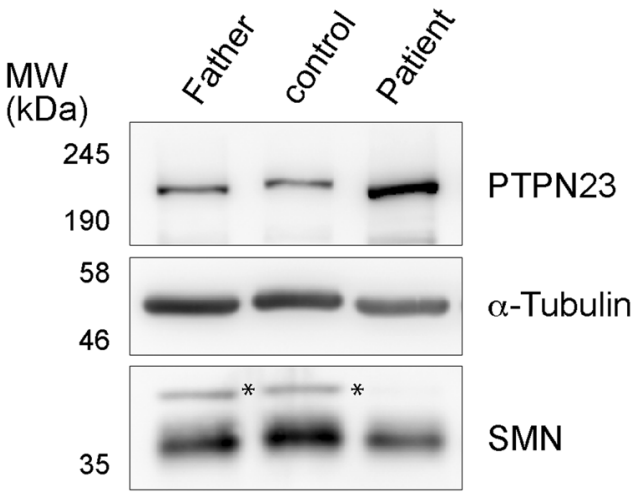

Fig. 4 Expression of PTPN23 in control fibroblast, patient fibroblast and fibroblasts from the patient's father was analysed by Immunoblot using specific antibodies against PTPN23 and compared to SMN. Detection of $\alpha$-tubulin served to equalize total protein amounts. The star $(*)$ indicates a slower migrating form of SMN that is lost in patient cells

SMN accumulation in CB, identified by the CB-marker coilin, was readily visible in more than $50 \%$ of all cells. In contrast, only $22 \%$ of patient fibroblasts showed respective SMN accumulations (Fig. $5 ; p=2.5 \times 10^{-7}$ vs. control cells and $p=1.9 \times 10^{-7}$ vs. parent cells; see Fig. S1 for additional examples in patient's cells). Cells still displaying SMN accumulations in patient fibroblasts regularly showed a weaker SMN signal than controls or parent cells. While we observed a reduction of SMN accumulation in $\mathrm{CB}$, the accumulation of coilin in $\mathrm{CB}$ was comparable in control cells, cells carrying the c.2974delC (p.(Leu992Tyrfs*168)) variant and patient cells (Fig. 6, see relative signal intensities of SMN and coilin in CB). The accumulation of coilin in $\mathrm{CB}$ indicates that the structure of $\mathrm{CBs}$ as a whole was not grossly affected in patient cells (Fig. S1, Figs. 5, Fig. 6). The loss of SMN from CB, however, regularly resulted in increased nucleoplasmic SMN in patient cells (Fig. 7, compare profiles in control and patient cells). These data show that compound heterozygous variants of PTPN23 abolish the efficient accumulation in SMN in CB. Similar observations were made previously after efficient knockdown of PTPN23 in human cells [7] consistent with the fact the PTPN23 variants described here strongly diminished the cellular function of PTPN23.

\section{Discussion}

We found two PTPN23 variants in a compound heterozygous state (c.2974delC (p.(Leu992Tyrfs*168)), c. 1902C >G (p.(Asn634Lys))) in a proband with severe and progressive neurological disease. The c.2974delC (p. (Leu992Tyrfs*168)) 1 bp deletion variant is most likely a loss of function (LOF) variant as it causes a frameshift predicted to result in considerable truncation of the 1637 amino acid long protein and/or nonsense mediated decay of the respective mRNA. The effect of the c.1902C $>\mathrm{G}$ (p. (Asn634Lys)) missense variant was predicted as deleterious by in silico analysis. Furthermore, important evidence for the functional defect of c.1902C $>\mathrm{G}$ (p.(Asn634Lys)) comes from the observation that patient's fibroblasts had reduced SMN localization in CB not only compared to wild type control but also to the cells of heterozygous carrier of the LOF PTPN23 variant (proband's healthy father with the c.2974delC (p.(Leu992Tyrfs*168)) variant).

Although the combination of alleles still allowed birth of a viable individual, the presented patient clinically shows a combination of severe developmental delay, epilepsy, hypomyelination and cortical blindness. Repeated MRI indicated that hypomyelination was followed by brain atrophy as evidenced by progressive damage of white matter as well as of the cerebral and cerebellar cortex. A primary microcephaly was noted as well. None of these manifestations were specific prompting us to describe the disease as a developmental and epileptic encephalopathy (according to ILAE) [14] with hypomyelination and brain atrophy.

Phenotypically similar patients were described by Alazami et al. [12], Trujillano et al. [13], and, most recently by Sowada et al. [15]. Alazami et al. reported a child with global developmental delay, epilepsy and brain atrophy associated with c.3995G $>\mathrm{T}$ (p.(Arg1332Leu)) homozygosity. Trujillano et al. [13] described a patient with global developmental delay, motor and speech delay, spasticity, seizures, microcephaly and developmental regression and brain atrophy, in whom homozygous c. $904 \mathrm{~A}>\mathrm{G}$ (p.(Met302Val)) variant were found. Functional studies were not performed, and Trujillano et al. suggested that recessive PTPN23 variant should be validated as being potentially causative for a specific phenotype including brain atrophy and developmental delay [13]. Sowada et al. [15] provided more clinical details on the subject originally reported by Alazami et al. [12] and described a third patient with a similar disease phenotype in whom compound heterozygous PTPN23 variants (c.3586C $>\mathrm{T}$ p. $(\operatorname{Arg} 1196 *)$, c.1595C $>$ T p.(Pro532Leu)) were found.

The displayed similarities of the phenotypes described in the literature and the present patient strongly support a causative link between PTPN23 defects and a specific disease. In search for cellular defects to better understand the mechanisms of pathogenicity, we studied patient's fibroblasts and evaluated the possibility that reduced PTPN23 activity caused a drop in SMN activity [7]. Indeed, we could demonstrate strongly reduced SMN accumulation in CB accompanied by higher nucleoplasmic levels of SMN. This suggests reduced UsnRNPs assembly activity despite similar levels of SMN expression [7]. Of note, our protein expression analysis of SMN revealed higher migrating 


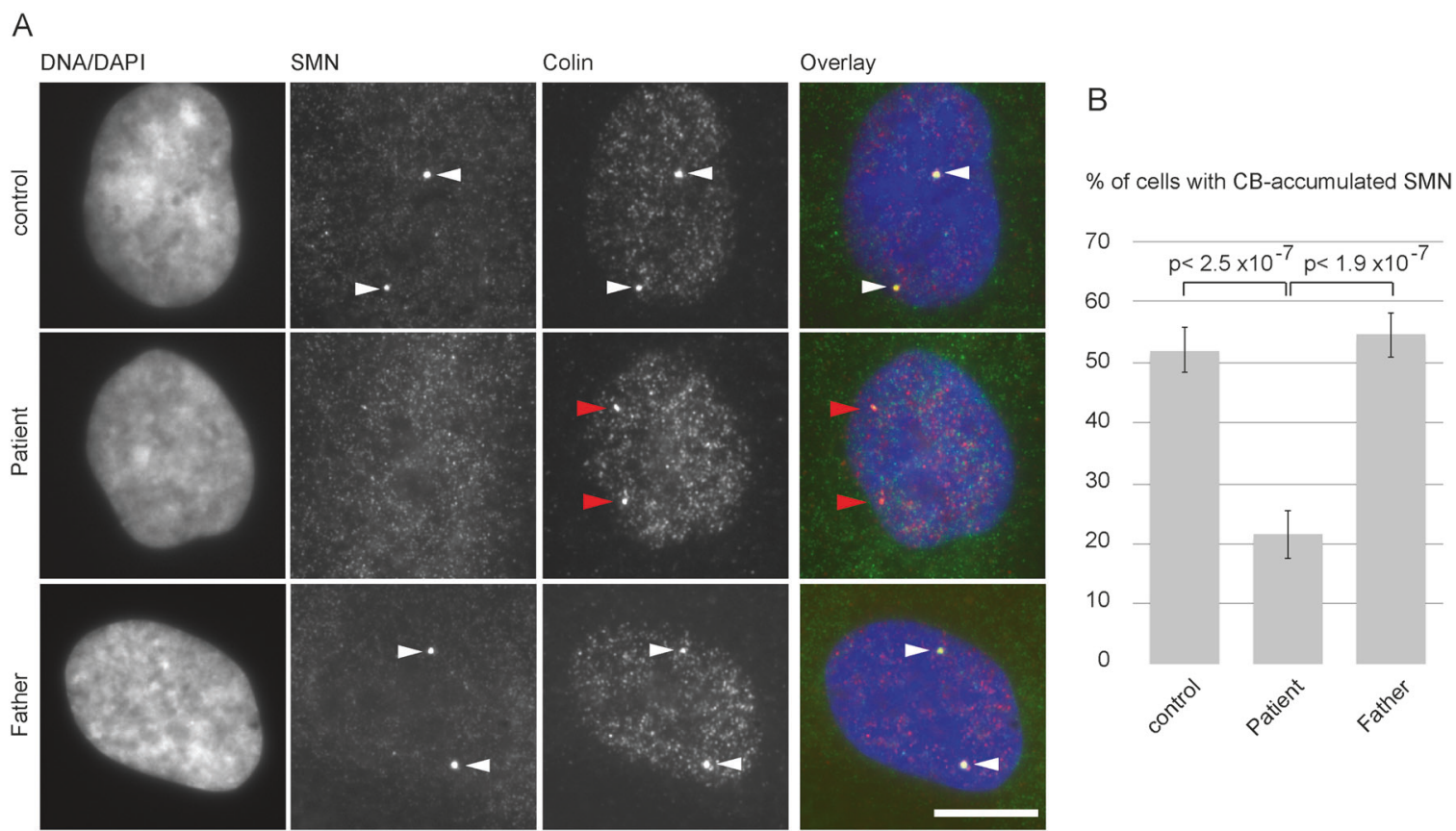

Fig. 5 a Immunofluorescence analysis of control fibroblasts, patient fibroblasts and fibroblasts from the patient's father. Antibodies against SMN and coilin (CB marker, red in overlay) were used to detect CB accumulations of SMN (green in overlay, see white arrowheads). Red arrowheads indicate CB accumulations of coilin without SMN accumulations. DAPI visualized cell nuclei (blue in overlay). Scalebar 20 $\mu \mathrm{m}$. b To quantify SMN accumulations in $\mathrm{CB}$, we determined the relative number $(N=100$ for control, unrelated as well as father fibroblasts) displaying at least one SMN accumulation in CB. Mean values from six independent experiments were calculated and significant differences determined using an unpaired student's $t$-test. The difference between the two situations was statistically significant with $p<2.5 \times 10^{-7}$ (control) or $p<1.9 \times 10^{-7}$ (father), respectively forms of SMN, indicative of modified SMN, only in controls cells but not in patient fibroblasts. This is consistent with reduced SMN phosphorylation as previously observed after the PTPN23 knockdown [7]. Despite the loss of one allele encoding full length PTPN23, the expression of fulllength PTPN23 was even higher in patient fibroblasts compared to controls. Expression of PTPN23 from the one remaining allele encoding full-length protein, thus, seemed to be up-regulated. Our observations suggest a cellular compensation mechanism in patient cells aiming at making up for the loss of fully functional PTPN23 by increased protein expression.

Our data leave open if cells of our patient or the previously described individuals display, in addition, defects in other defined PTPN23 functions, in particular in endosomal cargo sorting, multivesicular body morphogenesis and cell migration. Of note, homozygous deletion of PTPN23 is lethal around day 9.5 in mice. In contrast, defects in brain development, e.g., after the loss of Otx2 (ref. [16]) generally result in later lethality in mice. This suggests that, in mice, PTPN23 serves (a) basic cellular function(s), the loss of which causes defects in early embryogenesis at a stage where many organs only start to be developed. Although these results do not allow conclusions about an essential function of PTPN23 in human development, one may still speculate that complete homozygous LOF in both PTPN23 alleles is lethal for human development as well. In turn, the consistent clinical symptoms observed in the present and previously described patients suggests hypomorphic PTPN23 alleles that may still allow some basic functions but, by no means, display regular PTPN23 activity. This conclusion is further supported by the fact that efficient knockdown (i.e., strongly reduced function) of PTPN23 leads to a similar loss in SMN accumulation in $\mathrm{CB}$ than observed here.

Reduced SMN function caused by variants in SMN1 is known to cause the progressive lesion of spinal motor neurons and SMA (spinal muscular atrophy), whereas the complete loss of function (of both SMN1 and SMN2) is lethal. The SMA symptoms vary in severity depending on the amount of functional SMN protein but they are dominated by hypotonia, progressive muscle weakness and flaccid paralysis. In addition to these well-known manifestations Harding et al. found that in SMA neuronal degeneration included not only anterior horn cells, dorsal root ganglia and thalamus but also the cerebral cortex, basal ganglia, pigmented nuclei, brainstem and cerebellum [17]. Additionally, in a mouse model of severe SMA, Wishart et al. showed that reduced levels of SMN impaired perinatal brain development [18]. 

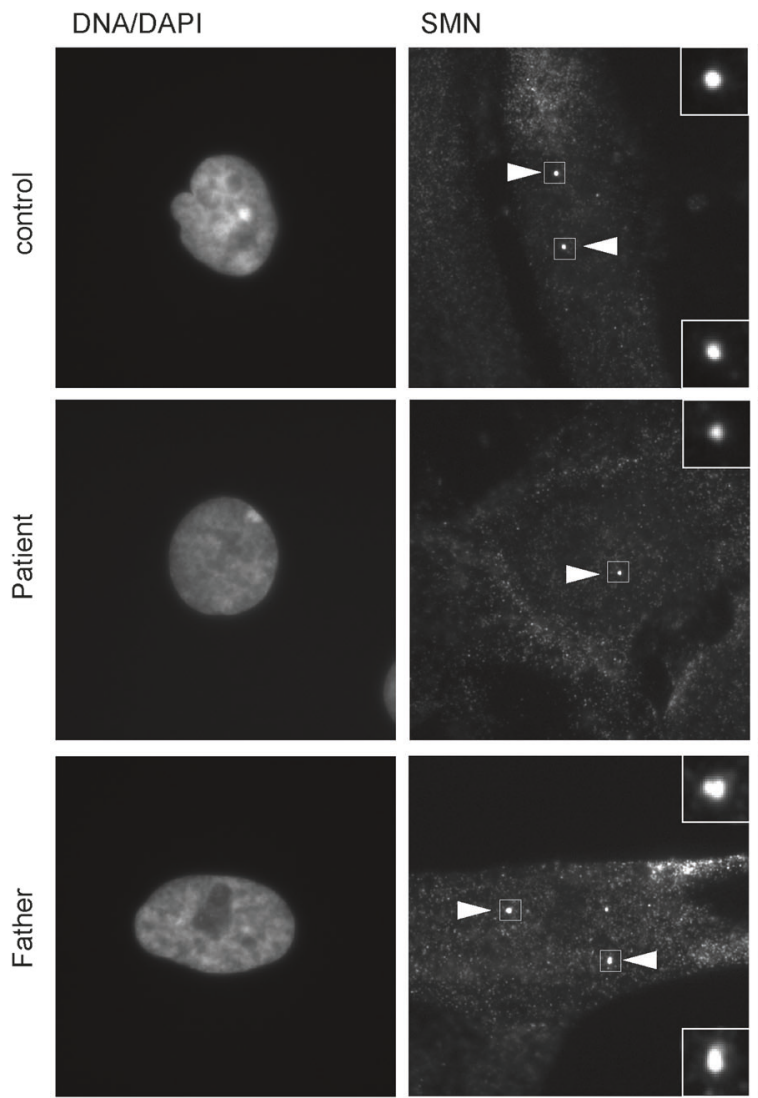

Fig. 6 Immunofluorescence analysis of control fibroblasts, patient fibroblasts and fibroblasts from the patient's father. Antibodies against SMN (green in overlay) and coilin (red in overlay) were used to detect

Except for the rather broadly appearing symptom of hypotonia in the newborn period, the phenotype of our patient (spasticity, developmental delay, epilepsy, hypomyelination, and brain atrophy) is considerably different than that typical for SMA. On the other hand, variants of the heterogenous nuclear ribonucleoproetin U (HNRNPU) have been associated with early onset seizures, severe intellectual disability, severe speech impairment, hypotonia and central nervous system abnormalities [9]. It is known that changes in HNRNPU activity impair the maturation of U2snRNP [19]. Our case and the latter example thus converge to the idea that changes in UsnRNP activity, resulting in globally altered splicing patterns, will severely alter brain development in humans.

Given the abovementioned extensive manifestations of severe SMA [17, 18] it could be speculated that an overlap between the PTPN23-associated disease and SMA does exist but is obscured by quick progression of encephalopathy in the PTPN23 disease, which masks the initially present hypotonia with other symptoms. Moreover, although our cellular readout for SMN function shows a strong correlation with the observed clinical phenotype, we do not know if the altered SMN activity pattern contributes
Coilin
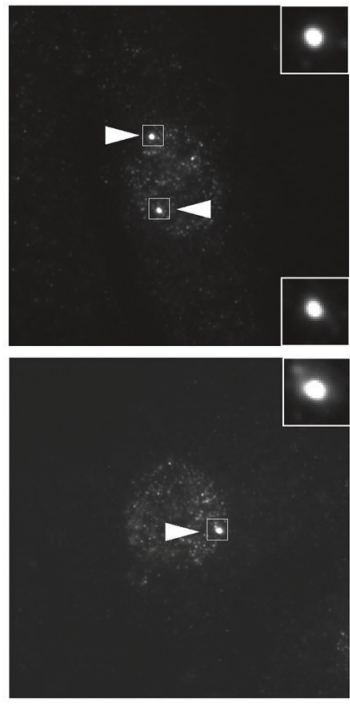

Overlay
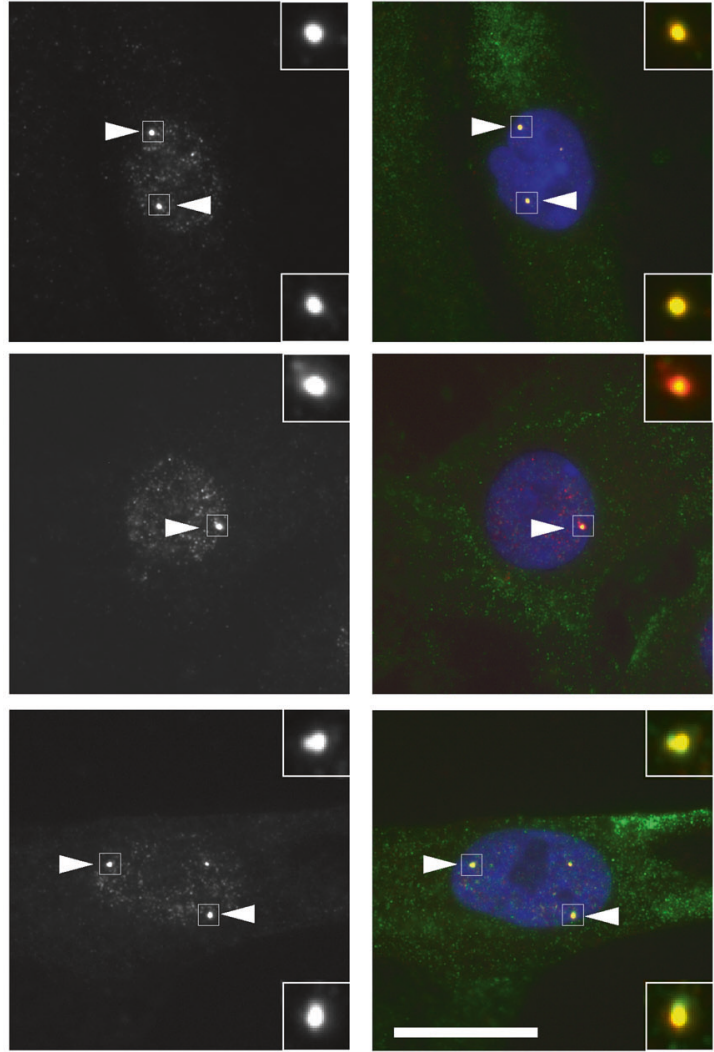

CB accumulations of SMN (see arrowheads and inlets). DAPI visualized cell nuclei (blue in overlay). Scalebar $20 \mu \mathrm{m}$

to the observed pathology. We consider it likely that the pathogenic mechanism of the disease in our subject will involve a combination of reduced cellular functions of the PTPN23 protein, including the regulation of SMN, but also several others.

In conclusion, we provide the first detailed description of a proband with novel compound heterozygous PTPN23 variants whose pathogenicity was substantiated by a functional study showing defects in SMN functions at the cellular level.

\section{Materials and methods}

\section{Patient}

We investigated a six-year-old girl who presented severe developmental and intellectual delay with nystagmus, vision loss, seizures, with microcephaly, spasticity and hypomyelination and brain atrophy in MRI. Additionally, we studied the healthy parents of the proband. Venous blood samples were collected from all the above mentioned participants of the study. Written informed consent was 

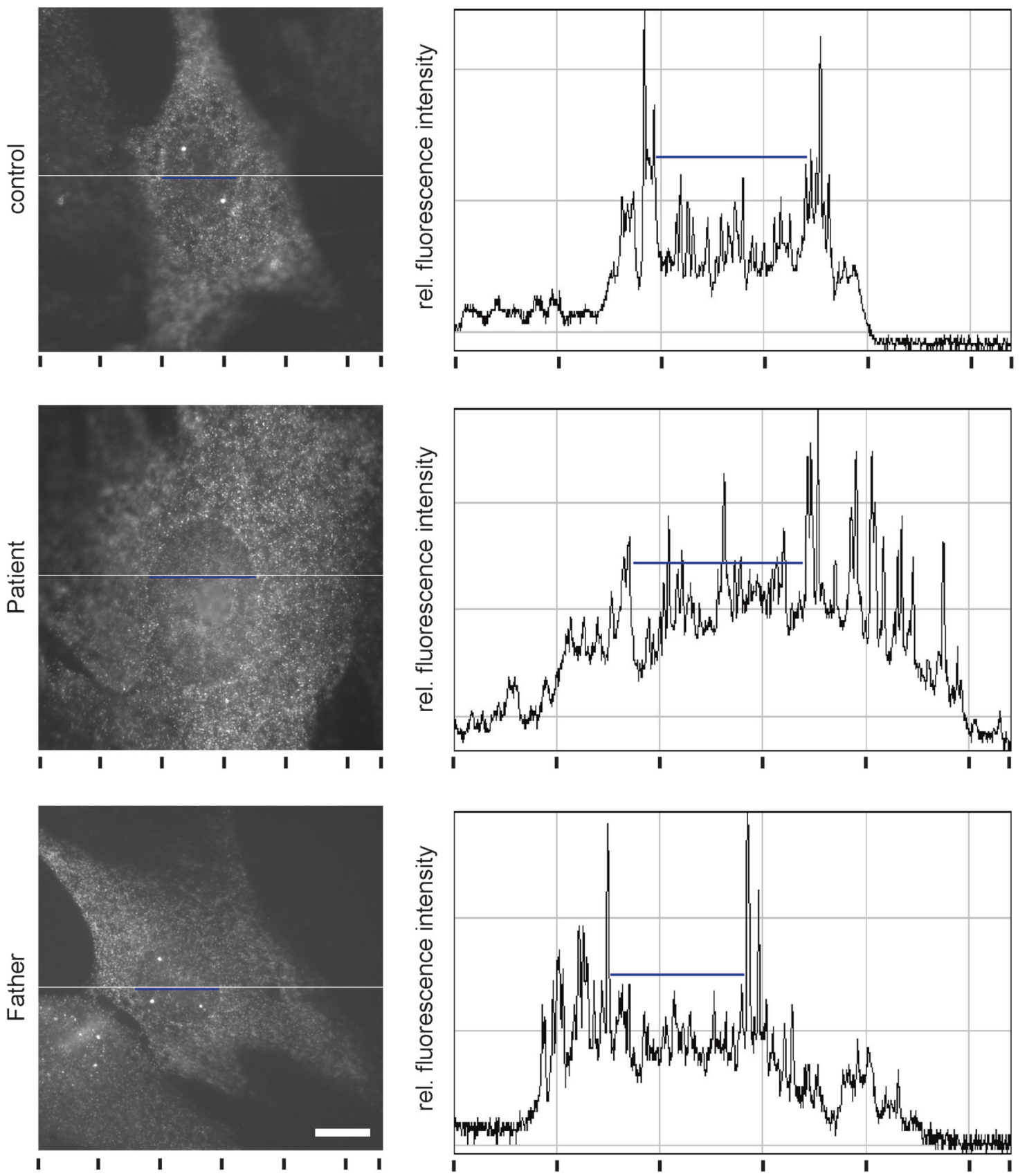

Fig. 7 Nucleo-cytoplasmic distribution of SMN. Immunofluorescence analysis of SMN in patient fibroblasts was performed as described in Fig. 5. White lines in images indicate the track of fluorescence lines mark the position of the nucleus along the profile as deduced from DAPI staining. Scalebar $20 \mu \mathrm{m}$. Graphs indicate the relative fluorescence values in all pixels along the white lines. Please see black marks underneath the images and graphs for corresponding positions

obtained prior to genetic testing from the parents (the legal guardians of the proband). Ethics approval was granted by the Institutional Review Board of Warsaw Medical University.

\section{Whole exome sequencing (WES)}

WES was performed in the proband (without parents) using Nextera XT DNA Library Preparation Kit (Illumina, San
Diego, CA) on HiSeq 1500 (Illumina). Bioinformatics analysis of WES data was performed using previously described pipeline [20].

The analysis of WES data was done as follows: after inspection for pathogenic variants described in HGMD, variants passing a default quality filter were filtered to include those with $<1 \%$ minor allele frequency in GnomAD and an in-house database of $>1000$ exomes from Polish population. Synonymous and deep intronic/non-coding variants were 
excluded. The remaining 370 variants were searched for biallelic variants consistent with autosomal recessive inheritance and monoallelic variants potentially causative of dominantly inherited diseases (autosomal or X-linked).

The mean depth of coverage in sequenced sample was $46 \times, 85 \%$ of target sequence was covered $\min 20 \times$ and $97 \%$ min 10x. In order to confirm the results from WES and to verify carrier state of the participants of the study direct sequencing by Sanger method was performed using BigDye $^{\mathrm{TM}}$ Terminator v3.1 Cycle Sequencing Kit (Applied Biosystems, Foster City, CA). Sequencing was carried out on ABI PRISM 3500 Genetic Analyzer (Applied Biosystems, Foster City, CA) and the results were analyzed with Variant Reporter 1.1 (Applied Biosystems). The following PCR primers were used for Sanger sequencing: c. 1902C $>$ G (p.(Asn634Lys)): 5'-TGC GTG AGC TTA TCC AGA AA3', 5'-AGC AGA GCA GCC ACC TTG -3'; c.2974delC (p.(Leu992Tyrfs*168)): 5'-CCA CCT CCT CAA TTC TCA GG 3', 5'-ACC AGG TGG GGA CTA GGG-3'.

The PTPN23 transcript NM_015466.2 is used for reference throughout the manuscript. Identified variants were submitted to Leiden Open Variation Database and Individual ID is 00151815 (https://databases.lovd.nl/shared/ individuals/00151815).

\section{Fibroblast cell culture}

Human fibroblasts were cultivated in 1:1 DMEM/F12 Medium (Thermo) supplemented with 15\% (v/v) FCS (fetal calf serum), $1 \%(\mathrm{v} / \mathrm{v})$ L-Glutamine, $1 \%$ Penicillin and $1 \%$ Streptomycine at $37{ }^{\circ} \mathrm{C}$ and $5 \% \quad \mathrm{CO}_{2}$. For immunofluorescence cells were grown on glass cover slips.

\section{Western blot}

Immunoblot analysis of SMN and PTPN23 was performed according to standard methods using a previously published PTPN23 antibody [7]. SMN was detected using a monoclonal mouse antibody against SMN (7B10, 1:500 a kind gift from Utz Fischer, Würzburg). Antibodies against $\alpha$ Tubulin (Sigma, T9026, 1:1000) were used to normalize protein contents on the blots.

\section{Indirect immunofluorescence}

For immunofluorescence, cells were grown on cover slips, washed with $1 \times$ PBS and fixed in $4 \%$ paraformaldehyde for $10 \mathrm{~min}$ at room temperature. Afterwards, cells were washed with $1 \times$ PBS and blocked using $1 \times$ PBS containing $0.1 \%$ Triton X100 and 10\% FCS (PBSTS). Fixed cells were incubated with $\alpha$-SMN (7B10, 1:100), and rabbit $\alpha$-coilin (Cell Signaling, D2L3J, 1:500) in the same buffer without FCS. DNA was stained using DAPI (4,6-diamidino-2- phenylindole) at a concentration of $0.25 \mu \mathrm{g} / \mathrm{ml}$. Cells were washed and mounted on object slides using Fluoromont-G mounting medium (Southernbiotech, Cambridge). Analysis was done on a Zeiss AXIOPHOT microscope using a $100 \times$ $1,3 \mathrm{NA}$ or $63 \times 1,4 \mathrm{NA}$ oil immersion objective. Images were processed using the ZEN2012 software and Photoshop CS5.

\section{Quantification of SMN accumulation in CB and image analysis}

To quantify SMN accumulations in CB, we determined the relative number ( $n=100$ for control, unrelated as well as father fibroblasts) displaying at least one SMN accumulation in CB. Mean values from six independent experiments were calculated and significant differences determined using an unpaired student's $t$-test. The nucleocytoplasmic distribution of SMN was determined using representative examples of SMN detection in the respective cells types. Profiles of fluorescence signals were determined using a straight line and the Plot Profile function in ImageJ64.

Acknowledgements The study was supported by the National Science Centre (NCN) Poland grant 2013/11/B/NZ7/04944 and the German Research Foundation (DFG), grant GR 1737/10-2. We would like to thank Utz Fischer, University of Würzburg, for anti-SMN antibodies and Walter Witke, University of Bonn for fruitful discussions.

\section{Compliance with ethical standards}

Conflict of interest The authors declare that they have no conflict of interest.

\section{References}

1. Gingras MC, Zhang YL, Kharitidi D, et al. HD-PTP is a catalytically inactive tyrosine phosphatase due to a conserved divergence in its phosphatase domain. PLoS One. 2009;4:e5105.

2. Doyotte A, Mironov A, McKenzie E, Woodman P. The Bro1related protein HD-PTP/PTPN23 is required for endosomal cargo sorting and multivesicular body morphogenesis. Proc Natl Acad Sci USA. 2008;105:6308-13.

3. Kim J, Lee JE, Heynen-Genel S, et al. Functional genomic screen for modulators of ciliogenesis and cilium length. Nature. 2010;464:1048-51.

4. Cao L, Zhang L, Ruiz-Lozano P, et al. A novel putative proteintyrosine phosphatase contains a BRO1-like domain and suppresses Ha-ras-mediated transformation. $J$ Biol Chem. 1998;273:21077-83.

5. Gingras MC, Kharitidi D, Chenard V, et al. Expression analysis and essential role of the putative tyrosine phosphatase Hisdomain-containing protein tyrosine phosphatase (HD-PTP). Int J Dev Biol. 2009;53:1069-74.

6. Manteghi S, Gingras MC, Kharitidi D, et al. Haploin sufficiency of the ESCRT component HD-PTP predisposes to cancer. Cell Rep. 2016;15:1893-1900.

7. Husedzinovic A, Neumann B, Reymann J, et al. The catalytically inactive tyrosine phosphatase HD-PTP/PTPN23 is a novel regulator of SMN complex localization. Mol Biol Cell. 2015;26:161-71. 
8. Krausova M, Stanek D. snRNP proteins in health and disease. Semin Cell Dev Biol. 2017. https://doi.org/10.1016/j.semcdb. 2017.10.011.

9. Bramswig NC, Ludecke HJ, Hamdan FF, et al. Heterozygous HNRNPU variants cause early onset epilepsy and severe intellectual disability. Hum Genet. 2017;136:821-34.

10. Bain JM, Cho MT, Telegrafi A, et al. Variants in HNRNPH2 on the $\mathrm{X}$ chromosome are associated with a neurodevelopmental disorder in females. Am J Hum Genet. 2016;99:728-34.

11. Gruss OJ, Meduri R, Schilling M, Fischer U. UsnRNP biogenesis: mechanisms and regulation. Chromosoma. 2017;126:577-93.

12. Alazami AM, Patel N, Shamseldin HE, et al. Accelerating novel candidate gene discovery in neurogenetic disorders via wholeexome sequencing of prescreened multiplex consanguineous families. Cell Rep. 2015;10:148-61.

13. Trujillano D, Bertoli-Avella AM, Kumar Kandaswamy K, et al. Clinical exome sequencing: results from 2819 samples reflecting 1000 families. Eur J Hum Genet. 2017;25:176-82.

14. Scheffer IE, Berkovic S, Capovilla G, et al. ILAE classification of the epilepsies: position paper of the ILAE Commission for
Classification and Terminology. Epilepsia. 2017;58:512-21. https://doi.org/10.1111/epi.13709. 2017/03/10

15. Sowada N, Hashem MO, Yilmaz R, et al. Mutations of PTPN23 in developmental and epileptic encephalopathy. Hum Genet. 2017;136:1455-61.

16. Boyl PP, Signore M, Annino A, Barbera JP, Acampora D, Simeone A. Otx genes in the development and evolution of the vertebrate brain. Int J Dev Neurosci. 2001;19:353-63.

17. Harding BN, Kariya S, Monani UR, et al. Spectrum of neuropathophysiology in spinal muscular atrophy type I. J Neuropathol Exp Neurol. 2015;74:15-24.

18. Wishart TM, Huang JP, Murray LM, et al. SMN deficiency disrupts brain development in a mouse model of severe spinal muscular atrophy. Hum Mol Genet. 2010;19:4216-28.

19. Xiao R, Tang P, Yang B, et al. Nuclear matrix factor hnRNP U/ SAF-A exerts a global control of alternative splicing by regulating U2 snRNP maturation. Mol Cell. 2012;45:656-68.

20. Ploski R, Pollak A, Muller S, et al. Does p.Q247X in TRIM63 cause human hypertrophic cardiomyopathy? Circ Res. 2014;114: e2-5. 